DOI: $10.2478 / v 10018-007-0004-6$

\title{
PERFORMANCE OF STATIC POSITIONING FOR MEDIUM DISTANCES BASED ON DATA FROM A VIRTUAL REFERENCE STATION AND ASG-PL NETWORK
}

\author{
M. Bakuła \\ University of Warmia and Mazury \\ Chair of Satellite Geodesy and Navigation \\ 5 Heweliusza St., 10-724 Olsztyn, Poland \\ e-mail: mbakula@uni.olsztyn.pl
}

\begin{abstract}
The use of a network of reference stations instead of a single reference station allows the modelling of some systematic errors in a region and allows a user to increase the distance between the rover receiver and reference stations. In some countries, GPS reference stations exist and GPS observations are available for users in real-time mode and in postprocessing. Observations from several GPS reference stations in a regional network enable modelling spatially-correlated errors and their modelling on an epoch-by-epoch and satelliteby-satellite basis. As a result, observations of a virtual reference station can be created at a rover's approximate position and its observations can be used in the precise baseline positioning of the rover. This paper presents the performance of the static positioning of a rover station, its quality and reliability for two different baselines. Single-baseline and network static solutions are presented and compared. Network solutions are based on data from a virtual reference station (VRS) obtained by the Wasoft/Virtuall software. In both cases, the same strategy of ambiguity resolution was used. These approaches have been tested with the use of 24-hour GPS data from the Polish Active Geodetic Network (ASG-PL). The data from three reference stations with medium-range separation were used in the process of generating VRS data. GPS data of the rover station were divided into 20, 10 and 5-min. sessions with a sampling interval of $5 \mathrm{sec}$. Practical calculations and analyses of horizontal and vertical accuracy of coordinates clearly show the improvement of static positioning in terms of time observation span and ambiguity reliability.
\end{abstract}

\section{INTRODUCTION}

With the establishment of active GPS reference networks with station distances of about one hundred kilometres or more, a new era of centimetre positioning has begun. Such networks have been established in many countries during recent years. Relative positioning will no longer be performed relative to one single reference station, but relative to several reference stations. 
The concept of using a multiple-reference station network for GPS positioning has been investigated by several research groups in recent years. A review of various multi-reference station network approaches can be found in Fotopoulos and Cannon (2001), Lachapelle and Alves (2002), Rizos (2002) and in the original papers (e.g., Wanninger, 1995; Wübbena et al., 1996; Wanninger, 1997, 1999, 2002, 2004; Raquet, 1997, 1997; Lachapelle et al., 2000; Euler et al, 2001; Landau et al., 2002; Grejner-Brzezińska et al., 2005). The presented models concern mostly carrier-phase positioning where high accuracy is expected, such as in $\mathrm{cm}$ or $\mathrm{mm}$. To achieve this, the ambiguity resolution process should be performed first.

Centimetre-level positioning in an array of reference station (RS) with a station spacing of about one hundred kilometres can be achieved using virtual reference station (VRS) observation (Wanninger, 2002). Precise correction models for dispersive (ionospheric) and non-dispersive (tropospheric and orbit) distance-dependent biases are obtained from the real reference data and used in the virtual observation calculations. This concept, in real-time positioning, is realised by setting up a central computing facility collecting the RS observation data and performing pre-processing to obtain correction models for distance-dependent biases. The user can transmit his approximate position to the central computing facility and by return he receives VRS observations based on the real RS data and the correction models (Euler et al, 2001; Landau et al., 2002). This concept has several advantages and allows observation errors to be reduced by error modelling in the network. This enables faster and more reliable ambiguity resolution and more accurate coordinates. Furthermore, redundant observations of several reference stations improves the reliability of the reference station data. In the end, the network of active reference stations makes use of temporary local reference stations at any position.

The reduction of differential ionospheric refraction effects is the most important step for the improvement of the ambiguity resolution. Modelling of distance-dependent errors using the observation data of several reference stations can only be performed successfully if the spatial extensions of disturbing futures are of larger scale than the station distances. With the station distances of some tens $\mathrm{km}$ this is always the case for the effects of orbit errors and also for a large portion of the tropospheric errors and large scale features of the ionospheric refraction (Wanninger, 2002).

The paper presents practical performance of static positioning in post-processing mode based on the Wasoft/Virtuall software for VRS generation by Wanninger. GPS data were used from active Polish network ASG-PL.

\section{OBSERVATIONS OF A VIRTUAL REFERENCE STATION COMPUTED BY WASOFT/VIRTUELL}

The installation of a temporary reference station in the area to be surveyed allows the calculation of shorter positioning baselines. Such temporary reference stations can be substituted by virtual reference stations (VRS) computed from the observations of the surrounding real reference stations. The VRS position can be freely selected. Its distance to the rover stations should be as short as possible.

One method to use the full information content of simultaneous observations of several reference stations is to combine them in such a way that an optimum set of code and carrier phase observations of a virtual reference station is obtained, which can then be used to determined the position of a rover receiver in baseline mode. Since some of the observation errors depend on the horizontal position differences of the rover receiver in relation to the reference stations, the rover's approximate position has to be known. The virtual reference station is assumed to be located at the rover's approximate position or in the vicinity. 
Several processing steps have to be performed in order to transform the carrier phase observations of the network of real reference stations to carrier phase observations of a virtual reference station (Wanninger, 1997). They include the resolution and removal of doubledifference carrier phase ambiguities. This step is a prerequisite for all further error modeling and reduction. If ambiguity fails for any observation, this observation has to be excluded from further data processing. Since the coordinates of the reference stations in the network are precisely known, ambiguity resolution is much simpler to perform than for unknown baselines. On the other hand, distance-dependent errors limit the complete ambiguity resolution to maximum distances between reference stations of 50 to $100 \mathrm{~km}$ (Wanninger, 2002). After the successful ambiguity removal, error models are calculated from the carrier phase observations. The ionospheric model is based on the ionospheric linear combination while the geometric model (which contains the tropospheric and orbit errors) is based on the ionosphere-free linear combination. The differential effects of these distance-dependent errors are modeled directly, i.e. no mapping to zenith direction is performed. The bilinear surface is used in Wasoft/Virtuell to describe the influence of errors and remove them. Each such plane is determined by two parameters: its inclination in two defined directions, e.g. north-south and east-west. The correction models are produced for each satellite and with a high temporal resolution. A minimum number of three reference stations are required.

The traditional static positioning of the rover stations can be performed using an appropriate VRS as reference. WaSoft/Virtuell computes VRS observations from the real observations of at least 3 GPS reference stations surrounding the selected VRS position. WaSoft/Virtuell uses the Receiver Independent Exchange Format (RINEX) as output format so that one can use any available post-processing software for GPS positioning.

\section{NUMERICAL EXAMPLE}

The GPS data used in this chapter were collected at four known permanent reference stations of the ASG-PL (e.g. Polish Active Geodetic Network) network (Fig. 1): LELO in Lelów, KATO in Katowice, WODZ in Wodzisław Śląski, and KRAW in Kraków, Poland (Kryński et al, 2003). The reference stations are equipped with the Ashtech $\mu Z$-CGRS (Continuous Geodetic Reference Station) receivers and ASH701945C_M SNOW antennas. The GPS observations were done on 1 March 2005 and lasted 24 hours (except data from 7:00 to 7:20 h and from 8:00 to 8:40 $\mathrm{h}$ due to gaps in GPS observations at the station KATO) and the sampling interval was 5 seconds. In the following calculations, the station KATO was treated as unknown whereas other three: WODZ, LELO and KRAW were treated as the reference stations and were used to generate the data of a VRS station.

Static positioning was elaborated as follows:

1) One-baseline solution: LELO-KATO (63 km), using real GPS data

2) Network VRS solution e.g. baseline VRS-KATO $(0 \mathrm{~km})$.

The rover station KATO is located nearly in the middle of the triangle LELO-KATOWODZ, whereby data of the VRS station were obtained. It was specially selected due to its position because all interpolation methods result in the closest reference station having the most influence on the values of interpolated errors and biases.

For the both approaches, real errors were calculated $(\mathrm{dN}, \mathrm{dE}, \mathrm{dH})$ as differences between the fixed 'true' coordinates of the reference station and coordinates obtained in successive sessions of relative static positioning.

In the presented calculations, the Lw/Lc processing was implemented based on the Ashtech Office Suite (AOS) software. In this processing strategy, the AOS software at first 
determines the wide line ambiguity ( $\mathrm{Lw}$ ) and afterwards fixes the narrow lane ambiguity (Ln) by processing the ionospherically free carrier observable Lc. After a successful fixing of Ln, the software outputs L1, L2, Lw, Ln and Lc fixed solutions (Ashtech, 1998).

In order to derive optimum phase ambiguities from the float solution, the AOS software applies a time-optimized search technique, which is defined on the 20 sigma value of the determined float ambiguity, for the solutions with the smallest sum of squares residual error. The value 20 is used as a factor to guarantee that the correct solution is within the search area.

Statistical testing is used to verify the correctness of the ambiguity resolution. First, a Chisquare is performed on the a posteriori variance of the residuals with a default of a $95 \%$ minimum probability. Second, a Fisher test is performed with the ratios of the variances of the second to the best fitting solution with a reliability of $99.99 \%$. The ability for a fixed ambiguity solution is also limited by the required minimum ratio of variances for the Fisher test. Ashtech Office Suite uses by default a value of 2.

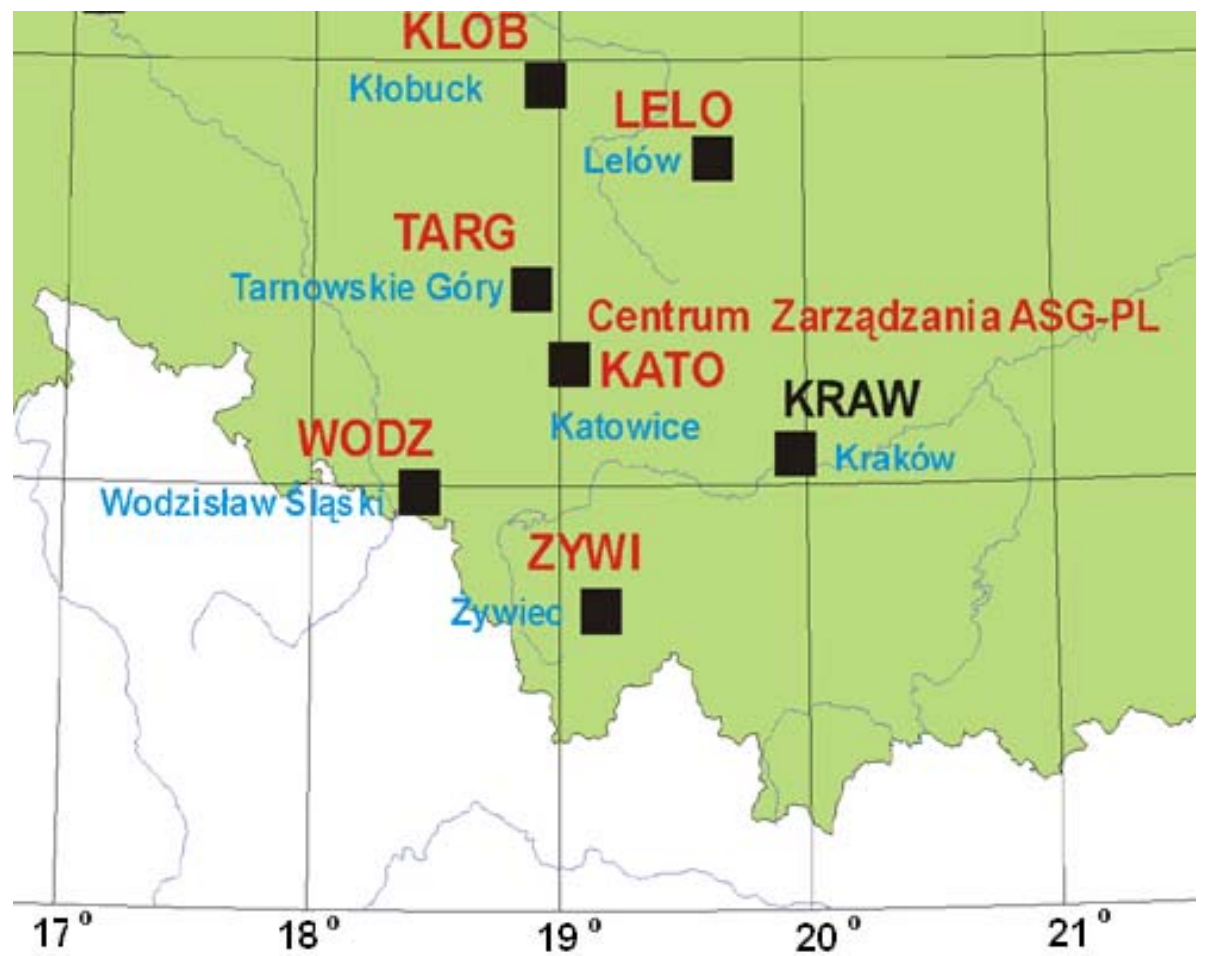

Fig. 1. Deployment of permanent reference stations in the southern part of Poland, as part of the ASG-PL network (the picture from the website www.asg-pp.pl/rozmieszczenie.html)

The results of 69 sessions in static positioning for the baseline LELO-KATO $(63 \mathrm{~km})$ in 20-min sessions are presented in Fig. 2. In these calculations there were a few baselines with gross errors caused by wrong ambiguity resolution i.e.

-for the session no 34 with errors $\mathrm{dN}=0.193 \mathrm{~m}, \mathrm{dE}=-2.247 \mathrm{~m}, \mathrm{dH}=1.131 \mathrm{~m}$; [12:00-12:20] -for the session no 48 with errors $\mathrm{dN}=0.897 \mathrm{~m}, \mathrm{dE}=1.974 \mathrm{~m}, \mathrm{dH}=0.913 \mathrm{~m}$; [16:40-17:00]

Excluding baselines with gross errors, there were 67 sessions for which the following statistics obtained: average errors of north, east and height components: $-0.007 \mathrm{~m}, 0.003 \mathrm{~m}$, $-0.094 \mathrm{~m}$ with standard deviations: $0.030 \mathrm{~m}, 0.025 \mathrm{~m}, 0.056 \mathrm{~m}$ respectively. Note, that the worst results are obtained for the height component. It should be noted that for most of the time, height errors have the same sign; it means that the systematic errors have occurred. 
In the case of the use of VRS data for the baseline VRS-KATO $(0 \mathrm{~km})$, there were no gross errors in the process of ambiguity resolution. The accuracy was as follows: average error of north, east and height positions: $-0.008 \mathrm{~m},-0.0004 \mathrm{~m},-0.002 \mathrm{~m}$ with standard deviations: $0.006 \mathrm{~m}, 0.005 \mathrm{~m}, 0.009 \mathrm{~m}$ respectively. It can be seen that the vertical positioning was extremely improved from about $10 \mathrm{~cm}$ to $1 \mathrm{~cm}$.

In the second stage of GPS elaboration, 69 twenty-minute sessions were divided into two each, so 138 new ten-minute sessions obtained. Results for the baseline LELO-KATO are presented in Fig 3. There were three sessions where gross errors were occurred:

-for the session no 24: $\mathrm{dN}=0.107 \mathrm{~m}, \mathrm{dE}=0.456 \mathrm{~m}, \mathrm{dH}=-0.172 \mathrm{~m}$; [03:50-04:00 h]

-for the session no 87: $\mathrm{dN}=0.075 \mathrm{~m}, \mathrm{dE}=1.171 \mathrm{~m}, \mathrm{dH}=-0.881 \mathrm{~m} ;[15: 20-15: 30 \mathrm{~h}]$

-for the session no 88: $\mathrm{dN}=-0.096 \mathrm{~m}, \mathrm{dE}=-3.055 \mathrm{~m}, \mathrm{dH}=-0.644 \mathrm{~m} ;[15: 30-15: 40 \mathrm{~h}]$

It should be noted that twenty-minute sessions number 34 and 38 in the first stage of elaboration had gross errors, but after dividing them into two separate ten-minute sessions the gross errors were vanished.
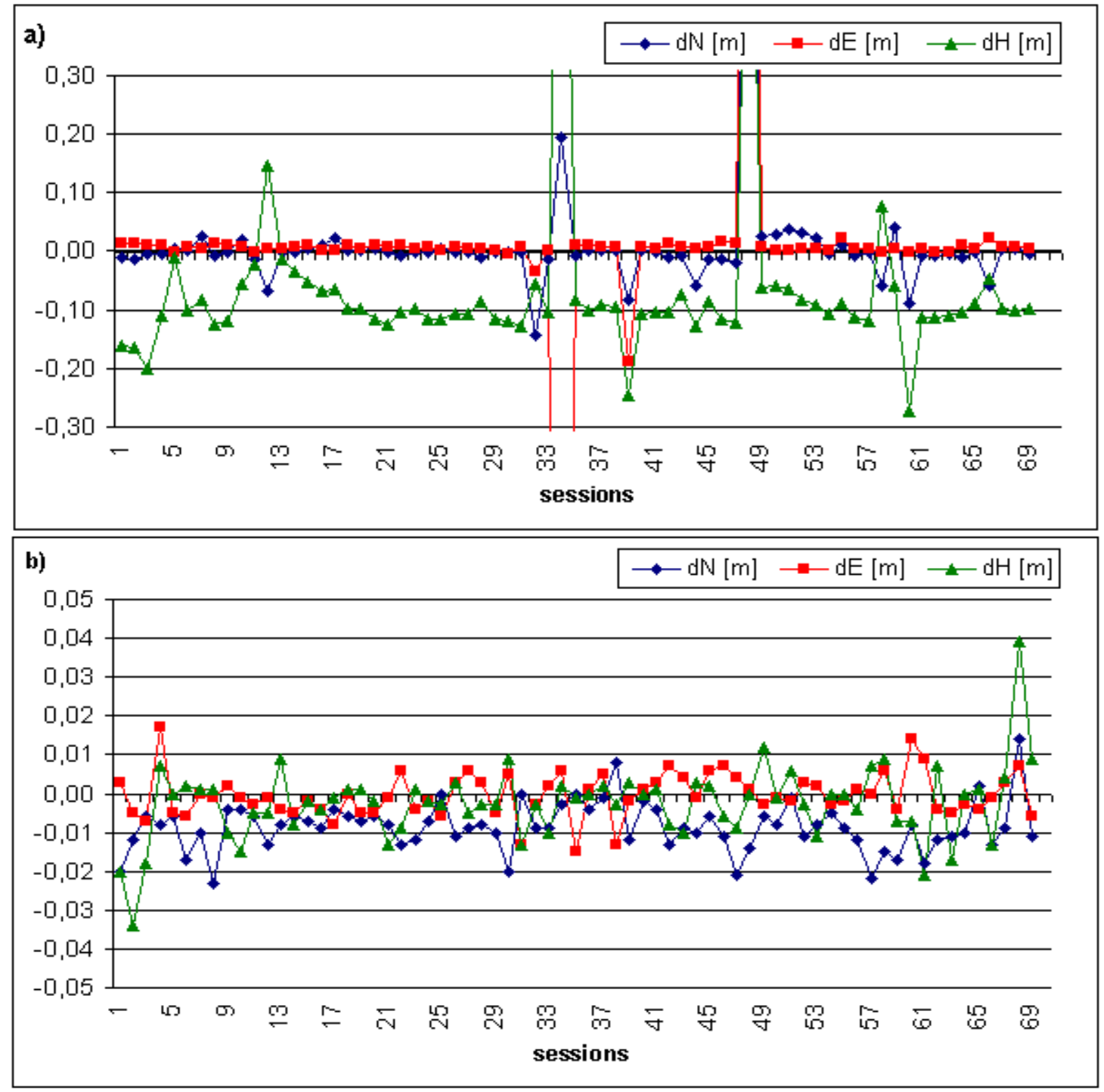

Fig. 2. Values of horizontal ( $\mathrm{dN} \mathrm{dE}$ ) and vertical $(\mathrm{dH})$ errors in static 20-min sessions positioning obtained in the local topocentric coordinate system:

a) for the baseline LELO-KATO, $(63 \mathrm{~km})$; b) for the baseline VRS-KATO, $(0 \mathrm{~km})$ 
Excluding baselines with gross errors, the following statistics were obtained for the baseline LELO-KATO; average errors of north, east and height components: $-0.007 \mathrm{~m}$, $0.002 \mathrm{~m},-0.099 \mathrm{~m}$ with standard deviations: $0.040 \mathrm{~m}, 0.024 \mathrm{~m}, 0.058 \mathrm{~m}$ respectively.

For the baseline VRS-KATO there were no gross errors and average errors of north, east and height components are as follows: $-0.007 \mathrm{~m}, 0.0003 \mathrm{~m},-0.003 \mathrm{~m}$ with standard deviations: $0.011 \mathrm{~m}, 0.007 \mathrm{~m}, 0.014 \mathrm{~m}$ respectively. As for ten-minute sessions in this case, the accuracy of the height component also improved.
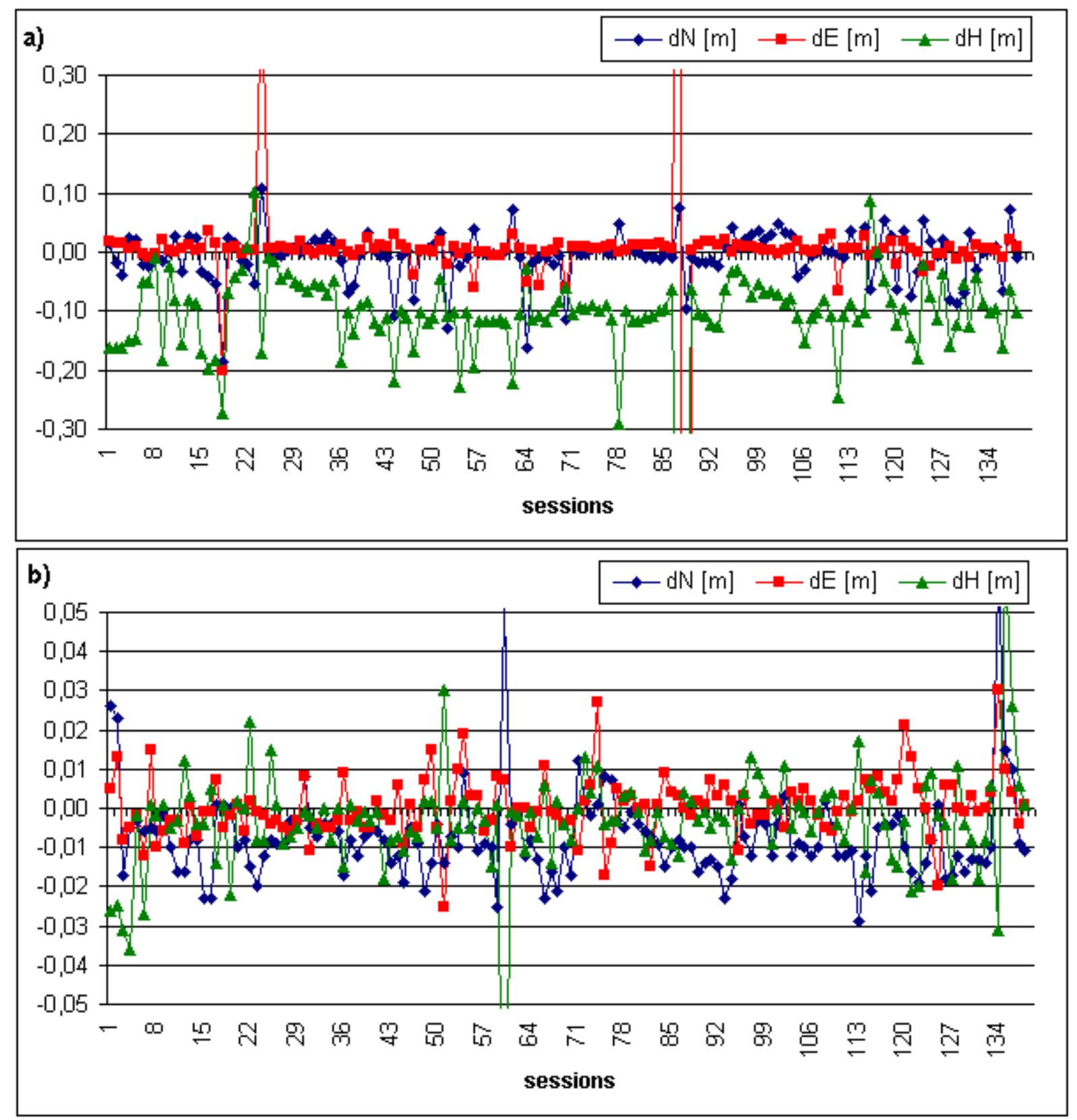

Fig. 3. Values of horizontal $(\mathrm{dN} \mathrm{dE})$ and vertical $(\mathrm{dH})$ errors in static 10-min sessions positioning obtained in the local topocentric coordinate system:

a) for the baseline LELO-KATO, $(63 \mathrm{~km})$; b) for the baseline VRS-KATO, $(0 \mathrm{~km})$

In the third stage of elaboration, the five-minute sessions contained only 60 epochs each. Results for the baseline LELO-KATO are presented in Fig 4. There were 12 baselines with 
gross errors. Real values of horizontal and vertical components, for more details, are presented in Table 1. Excluding sessions with gross errors, there were 264 sessions for the baseline LELO-KATO with proper ambiguity resolutions, for which the following statistics were obtained: average errors of coordinates: $-0.008 \mathrm{~m}, 0.001 \mathrm{~m},-0.091 \mathrm{~m}$ with standard deviations: $0.045 \mathrm{~m}, 0.023 \mathrm{~m}, 0.077 \mathrm{~m}$ respectively. However, for the baseline VRS-KATO there were only three baselines with gross errors:

- for the session no $125: \mathrm{dN}=0.480 \mathrm{~m}, \mathrm{dE}=-5.160 \mathrm{~m}, \mathrm{dH}=2.057 \mathrm{~m} ;[11: 20-11: 25 \mathrm{~h}]$

- for the session no 126: $\mathrm{dN}=-0.461 \mathrm{~m}, \mathrm{dE}=5.140 \mathrm{~m}, \mathrm{dH}=-1.898 \mathrm{~m} ;[11: 25-11: 30 \mathrm{~h}]$

- for the session no 141: $\mathrm{dN}=0.697 \mathrm{~m}, \mathrm{dE}=0.815 \mathrm{~m}, \mathrm{dH}=0.344 \mathrm{~m} ;[11: 40-11: 45 \mathrm{~h}]$
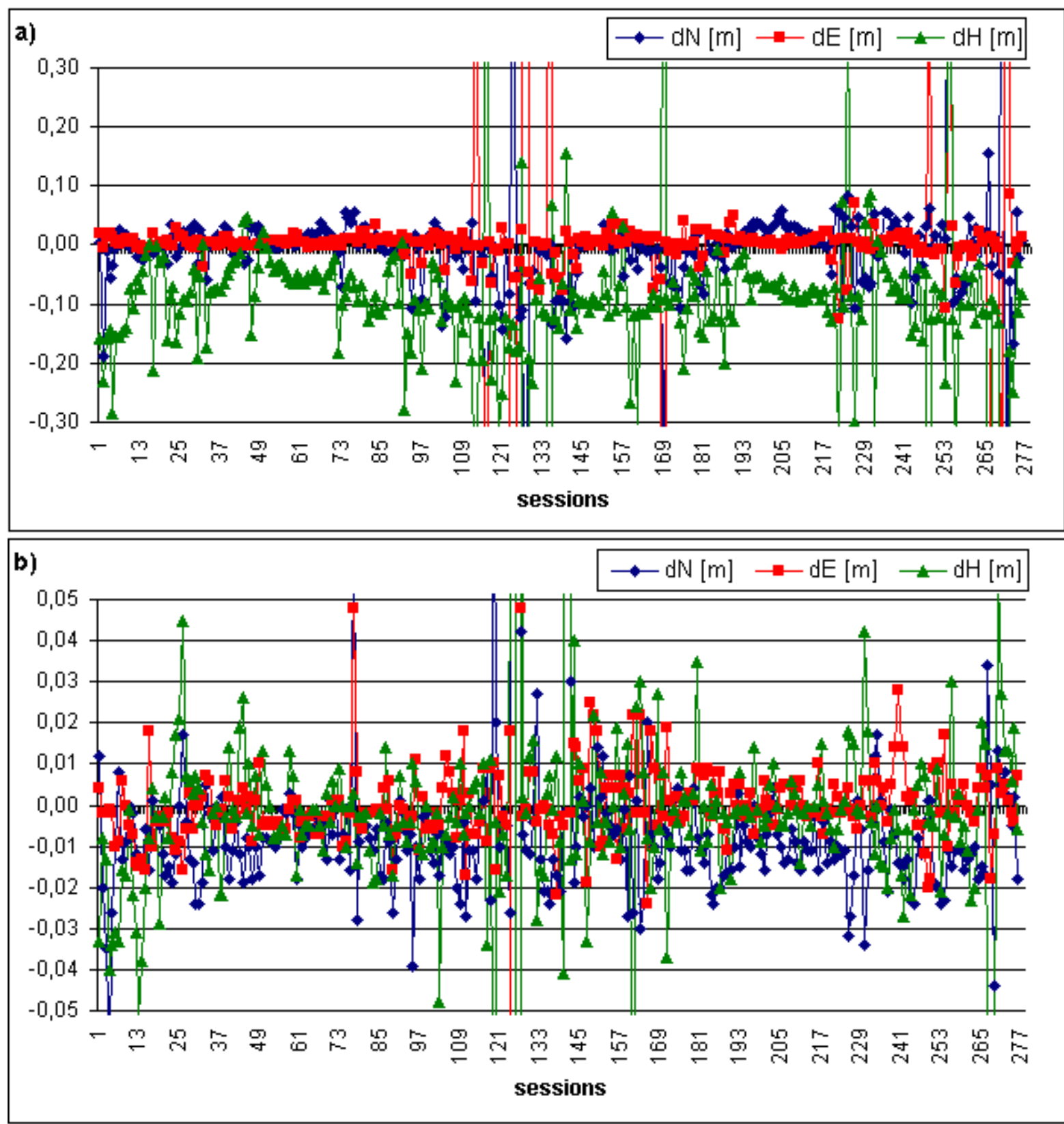

Fig. 4. Values of horizontal $(\mathrm{dN} \mathrm{dE})$ and vertical $(\mathrm{dH})$ errors in static 5-min sessions positioning obtained in the local topocentric coordinate system:

a) for the baseline LELO-KATO, $(63 \mathrm{~km})$; b) for the baseline VRS-KATO, $(0 \mathrm{~km})$

Note that for the sessions 125 and 126 with gross errors for the baseline VRS-KATO, there were similar observation conditions, but the true errors have almost the same absolute 
value but opposite signs. This shows that the statistical tests of ambiguity resolutions are not reliable. Excluding the sessions with gross errors, there were 273 good sessions with statistics such as average errors of north, east and height coordinates: $-0.008 \mathrm{~m}, 0.0003 \mathrm{~m},-0.003 \mathrm{~m}$ with standard deviations $0.013 \mathrm{~m}, 0.009 \mathrm{~m}, 0.023 \mathrm{~m}$ respectively. Additionally, the sessions 125, 126 and 141 for the baseline VRS-KATO were post-processed by the OTF (On The Fly) solutions, but the calculations were not finished successfully. It was good news concerning validation of OTF solutions. The OTF ambiguities were not fixed so the positions could not be treated seriously. From a practical point of view, the most dangerous situation is when the ambiguity process performs successfully according to the statistical tests, but in reality there are gross errors in positioning. The ambiguity searching criterion is just a statistical criterion and is not guaranteed in all applications (Xu, 2003).

Table 1.

\begin{tabular}{|c|c|c|c|c|}
\hline Session & $\mathbf{d N}[\mathbf{m}]$ & $\mathbf{d E}[\mathbf{m}]$ & $\mathbf{d H}[\mathbf{m}]$ & $\begin{array}{c}\text { Local Time } \\
\text { of observations }\end{array}$ \\
\hline 113 & $-0,095$ & 0,612 & $-0,562$ & $10: 20-10: 25$ \\
\hline 116 & $-0,639$ & $-0,800$ & 0,669 & $10: 35-10: 40$ \\
\hline 124 & 0,656 & $-1,510$ & $-0,135$ & $11: 15-11: 20$ \\
\hline 128 & $-0,542$ & 5,161 & $-1,789$ & $11: 35-11: 40$ \\
\hline 135 & 0,003 & 1,184 & $-1,184$ & $12: 10-12: 15$ \\
\hline 169 & $-0,422$ & $-4,421$ & 1,207 & $15: 00-15: 05$ \\
\hline 248 & 0,061 & 0,524 & $-2,023$ & $21: 30-21: 35$ \\
\hline 254 & 1,084 & 0,675 & 1,325 & $22: 05-22: 10$ \\
\hline 256 & $-0,055$ & $-0,065$ & $-0,363$ & $22: 15-22: 20$ \\
\hline 266 & 0,154 & $-0,742$ & $-1,705$ & $23: 05-23: 10$ \\
\hline 270 & 1,027 & $-0,986$ & $-1,092$ & $23: 25-23: 30$ \\
\hline 271 & $-0,532$ & 3,062 & $-0,973$ & $23: 30-23: 35$ \\
\hline
\end{tabular}

By analysing local time in which the gross errors occurred for particular sessions, it was seen that in most cases there were rising satellites. The influence of rising satellites on ambiguity resolution is known and has been investigated by many researchers and still more research is required in terms of ambiguity validation procedure.

\section{CONCLUSIONS}

In this paper, short-time static GPS positioning has been investigated based on the data from a virtual reference station and data from dual frequencies GPS receivers. Test data from an ASG-PL network were used to evaluate the performance of static positioning with the use of VRS data. The numerical results show that the use of VRS data for medium baselines can reduce distance-dependent errors in GPS positioning.

The success of precise static positioning depends on the ability to resolve integer phase ambiguities when short observation time is required. Although there were three gross errors for the baseline VRS-KATO $(0 \mathrm{~km})$ during five-minute sessions, this still represented $98,8 \%$ effectiveness. For the sessions of 10 and 20-minute of observations, the effectiveness was $100 \%$ whereas in the case of single baseline for the medium distance positioning there were not only gross errors, but also evident deterioration of vertical components. Applying the VRS data in static positioning improved accuracy, especially in improving height positioning from about $10 \mathrm{~cm}$ to $1 \mathrm{~cm}$.

The results presented in this paper show that post-processed baseline, for medium distances, based on one reference station had bias errors in the height component. It was overcome by the use of VRS data where three reference stations were used in the generating 
of the VRS station in the vicinity of the rover station. In this case, accuracy of vertical positioning was at the same level as the horizontal components, i.e. about $1 \mathrm{~cm}$, even for short 5-min static sessions.

\section{ACKNOWLEDGEMENTS}

The post-processing software Wasoft/Virtuell, generating VRS data, tested in this paper was kindly provided by Professor Lambert Wanninger. The author would like to express great gratitude to the Professor for allowing free testing of the VRS approach with the use of ASGPL network. The Head Office of Geodesy and Cartography, Poland and the Department of Geodesy and Cartography of the Province of Silesia kindly supplied GPS data from ASG-PL.

\section{REFERENCES}

Ashtech and Spectra Precision Terrasat GmbH Germany, (1998). Ashtech Office Suite for Survey, User's Manual, USA.

Euler H.J., Keenan C.R., Zebhauser B.E., Wübbena G. (2001). Study of Simplified Approach in Utilizing Information from Permanent Reference Station Arrays, Paper presented at ION GPS 2001, Salt Lake City, Utach, pp. 379-391.

Fotopoulos G., Cannon M.E. (2001). An Overview of Multi-Reference Station Methods for Cm-Level Positioning, GPS Solutions, Vol. 4,No. 3, pp. 1-10.

Grejner-Brzezińska D.A. Kashani I., Wielgosz P. (2005). On accuracy and reliability of instantaneous network RTK as a function of network geometry, station separation, and data processing strategies, GPS Solution, Vol. 9, N. 3, pp. 212-225.

Kryński J., Rogowski J.B., Zieliński J.B. (2003). National Report of Poland to EUREF 2003, EUREF Symposium of the IAG Subcommission for Eurpoe (EUREF) held in Toledo, Spain, 4-7 June 2003, EUREF publication No 12, Mitteilungen des Bundesamtes für Kartographie und Geodäsie, Band 33, Frankfurt am Main. Pp. 264-268.

Lachapelle G., Alves P., Fortes L.P., Cannon M.E., Townsend B. (2000): DGPS RTK Positioning Using a Reference Network, Proceedings of GPS2000 (Session C3, Salt Lake City, 19-22 September), The Institute of Navigation, Alexandria, VA, pp. 1165-1171.

Lachapelle G., Alves P. (2002): Multiple Reference Station Approach: Overview and Current Research, , Journal of Global Positioning Systems, Vol. 1, No 2, pp 133-136.

Landau H., Vollath U., Chen X. (2002). Virtual Reference Stations Systems, Journal of Global Positioning Systems, Vol. 1, No 2, pp 137-143.

Raquet J. (1997). A New Approach to GPS Carrier Phase Ambiguity Resolution Using a Reference Receiver Network, Proceedings of National Technical Meeting, Santa Monica, January 14-16), The Institute of Navigation, Alexandria, VA, pp. 357-366.

Raquet J. (1999). Development of a Method for Kinematic GPS Carrier Phase Ambiguity Resolution Using Multiple Reference Receiver, UCGE No 20116, (PhD thesis).

Rizos C. (2002). Network RTK Research and Implementation - A Geodetic Perspective, Journal of Global Positioning Systems, Vol. 1, No 2, pp 144-150.

Wanninger L. (1995). Improved Ambiguity Resolution by Regional Differential Modelling of the Ionosphere, Proceedings of ION GPS-95, Palm Springs, Sep. 12-15, pp 55-62. 
Wanninger L. (1997). Real-Time Differential GPS-Error Modelling in Regional Reference Station Networks in: Brunner, F.K. (Hg.): Advances in Positioning and Reference Frames, Proceedings of the IAG Scientific Assembly, Rio de Janeiro, Brazil, pp. 86-92.

Wanninger L. (1999). The Performance of Virtual Reference Stations in Active Geodetic GPS-Networks under Solar Maximum Conditions, Proc. of ION GPS 99, Nashville TN, pp.1419-1427.

Wanninger L. (2002). Virtual Reference Stations for Centimeter-Level Kinematic Positioning, Proc. of ION GPS 02, Portland, Oregon, pp.1400-1407.

Wanninger L. (2004). Ionospheric Disturbance Indices for RTK and Network RTK Positioning, Proc. of ION GNSS 2004, Long Beach, CA, pp. 2849-2854

Wübbena G., Bagge A., Seeber G., Böder V., Hankemeier P. (1996). Reducing Distance Dependent Errors for Real-Time Precise DGPS Applications by Establishing Reference Station Networks, Proceedings of the International Technical Meeting, ION GPS-96, Kansas City, Missouri, pp. 1845-1852.

$\mathrm{Xu}$ G. (2002). A General Criterion of Integer Ambiguity Search, Journal of Global Positioning Systems, Vol. 1, No 2, pp 122-131.

Received: 2006-01-11,

Reviewed: 2005-02-07, by W. Góral,

Accepted: 2006-02-27. 